\title{
Long non-coding RNA LINC00662 facilitates bladder cancer progression through modulating miR-195- 5p/VEGFA/Ras/Raf/MEK/ERK signaling pathway
}

Hengbing Wang

Huaian First People's Hospital

Xiaobing Niu

Huaian First People's Hospital

Hesong Jiang

Huaian First People's Hospital

Bing Zhong

Huaian First People's Hospital

Xi Jiang

Huaian First People's Hospital

Guangbo Fu ( $\sim$ guangbo_fu@163.com )

The affiliated huaian No.1 People's hospital of Nanjing medical university

Research

Keywords: Bladder cancer, LINC00662, LncRNA, MiR-195-5p, VEGFA

Posted Date: March 18th, 2020

DOl: https://doi.org/10.21203/rs.3.rs-17620/v1

License: (9) (i) This work is licensed under a Creative Commons Attribution 4.0 International License.

Read Full License 


\section{Abstract}

Background: Bladder cancer (BC) is one of the most common malignant tumors in the urinary system. Long non-coding RNA (IncRNA) plays an important role in BC.

Methods: LINC00662 expression was identified by quantitative real-time polymerase chain reaction (qPCR) and in situ hybridization (ISH). The effect of LINC00662 on cell proliferation, apoptosis, migration and invasion was measured by CCK8 assay, colony formation assay, transwell assay, and western blot. Dual-luciferase reporter gene assay, RNA pull-down and RIP assay confirm the interaction between LINC00662 and miR-195-5p/VEGFA. The in vivo effect of LINC00662 on BC was investigated using a mouse tumorigenicity model.

Results: LINC00662 was overexpressed in BC tissues and cell lines, and negatively correlated with the survival of BC patients. Overexpression of LINC00662 promoted proliferation, migration and invasion and inhibited apoptosis of BC cells, and LINC00662 knockdown abrogated this effect. Silencing LINC00662 inhibited BC growth in a subcutaneous BC tumor mouse model. LINC00662 acted as an oncogene through regulating miR-195-5p/VEGFA axes. Decreased VEGFA expression caused by LINC00662 knockdown inhibited the phosphorylation of Raf-1, MEK1/2, and ERK1/2, while this regulation effect was abrogated by miR-195-5p inhibitor.

Conclusion: LINC00662 regulated the proliferation, apoptosis, migration, and invasion of BC cells probably via miR-195-5p-mediated VEGFA/Ras/Raf/MEK/ERK signaling pathway.

\section{Background}

Bladder cancer (BC) is a malignant tumor that occurs in the bladder mucosa and is the most common malignant tumor in the urinary system. According to the updated cancer statistics in 2017, there were 79 030 cases and 16870 deaths of urinary $B C$ were estimated which is the sixth most common cancer and the eighth leading cause of death due to cancer in the United States ${ }^{1}$. The latest cancer statistics in 2015 for China showed that there were about 80500 new cases of BC, including 62100 males and 18400 females were diagnosed. The incidence of $\mathrm{BC}$ has surpassed prostate cancer and become the first urinary malignancies in male ${ }^{2}$. Although most patients have non-muscle invasive carcinoma at the initial diagnosis, these tumors are prone to recurrence, infiltration and drug resistance after treatment, which becomes a huge challenge for clinical treatment. Today, the survival rate of some cancer patients such as kidney cancer and prostate cancer has been improved obviously, however, the five-year survival rate of BC is still maintaining at a low level. Until recently, the treatment for $B C$ had seen little progress ${ }^{3}$. The pathobiological mechanisms of $\mathrm{BC}$ are still unclear. Therefore, exploring biomarkers or new therapeutic targets will facilitate the research of BC treatments.

LncRNA is a subgroup of non-coding RNAs consisting of more than 200 nucleotides originally considered to be by-products of RNA polymerase II transcription and do not have any biological function ${ }^{4}$. Recent 
studies show that IncRNA participates in many important regulatory processes in terms of epigenetic, transcriptional and post-transcriptional regulation, and IncRNA is becoming one of the hotspots in the field of oncology. Many studies have shown that abnormal expression of IncRNA exists in various tumors such as liver cancer, lung cancer, breast cancer, skin adenocarcinoma, glioma and gastric cancer ${ }^{5}$. The microRNA (miRNA) has been widely demonstrated to be involved in BC development, however, the biological function and regulatory role of IncRNA in BC are still unclear ${ }^{6}$. In recent years, the role of IncRNA in tumorigenesis, metastasis, and progression has attracted extensive concern ${ }^{7,8}$. Studies have reported that several IncRNAs such as UCA1, MALAT1, ncRNA, GAS5, and $\mathrm{H} 19$ are involved in the development and progression of $\mathrm{BC}^{9,10}$.

LINC00662 has been shown to be abnormally expressed in lung cancer ${ }^{11}$, gastric cancer ${ }^{12}$, and oral squamous cell carcinoma ${ }^{13}$, and plays a regulatory role in the development of these tumors. It has already shown its potential value in early diagnosis, and it will be more promising to become a new target for cancer treatment in the future. At present, the role of LINC00662 in BC has also attracted people's attention, but its biological function and regulatory role still need to be further revealed.

In this study, we investigated the expression of LINC00662 in BC and its regulatory effect and potential mechanisms in BC development and progression. Our results suggested that LINC00662 is up-regulated in $\mathrm{BC}$ tissues and $\mathrm{BC}$ cell lines, and its high expression promotes the proliferation, migration, and invasion activities of BC cells in vitro and promotes tumor growth in vivo. Inhibition of LINC00662 expression showed an anti-tumor effect. Overall, our study indicated that LINC00662 plays a regulatory role in development and invasion of BC through miR-195-5p-mediated VEGFA/Ras/Raf/MEK/ERK signaling pathway, and the LINC00662 may be a potential biomarker and target for BC.

\section{Materials And Methods}

Cell lines and animals

The human bladder epithelium immortalized cell line SV-HUC-1 and BC cell lines T24, RT4, 5637, J82, and SW780 were purchased from ATCC (MD, USA). The cells were cultured in DMEM containing $10 \%$ fetal bovine serum in an incubator with $5 \% \mathrm{CO}_{2}$ at $37^{\circ} \mathrm{C}$.

Babl/c mice (20 24 g, 6 weeks) were purchased from Beijing HFK Bioscience Co. Ltd. (Beijing, China). The animals were housed in a laboratory with temperature control of $24 \pm 2{ }^{\circ} \mathrm{C}$ and a humidity of $40 \% \sim$ $60 \%$. The animal experiment was approved by the medical ethics committee of The Affiliated Huaian No.1 People's Hospital of Nanjing Medical University.

BC tissue sample

Sixty clinical BC tissues and paired paracancerous tissues were from the resected specimens of BC patients from January 2006 to May 2012 in the Affiliated Huaian No.1 People's Hospital of Nanjing Medical University. The samples were confirmed pathologically by two pathologists independently and 
stored in liquid nitrogen. This study was approved by the Institutional Ethical Review Committee of the Affiliated Huaian No.1 People's Hospital of Nanjing Medical University. All patients enrolled in the study signed written informed consent.

Vectors construction and transfection

Over-expression of LINC00662 and VEGFA were achieved by using a pcDNA3.1 vector (GenePharma, Shanghai, China) transfection. The shRNA against LINC00662 (5'-GTGATTCATACATCCCTATGG-3') was synthesized to knockdown of LINC00662 (Gene-Pharma) according to the BLOCK-iT ${ }^{\mathrm{TM}}$ RNAi Designer (Thermo). The miR-195-5p mimics and miR-195-5p inhibitor were designed and synthesized by GenePharma. The empty vectors were used as control. Lipofectamine ${ }^{T M} 2000$ kit was used for transfection operations.

Cell proliferation activity

Cell proliferation was measured using the CCK-8 kit (Beyotime). Briefly, after transfection, the cells were digested and collected, washed with PBS, and then resuspended in DMEM medium to $5 \times 10^{4} \mathrm{cell} / \mathrm{s} / \mathrm{ml}$. The cell suspensions were added to a 96 -well culture plate at $100 \mu \mathrm{l} /$ well. The cells were incubated for 24 , 48 and $72 \mathrm{~h}$, respectively, and then $10 \mu \mathrm{l}$ of CCK-8 solution was added to each well and incubated for $2 \mathrm{~h}$. The absorbance was determined at $450 \mathrm{~nm}$ using an Infinite M200 spectrophotometer (TECAN). Six duplicates for each group.

TUNEL staining assay

An in situ cell death detection kit (Roche) was conducted to measure the apoptosis. Briefly, after transfection, the cells were digested and collected, washed with PBS, and then resuspended in DMEM medium to $5 \times 10^{4}$ cells $/ \mathrm{ml}$. The cell suspensions were added to a 24 -well culture plate at $500 \mu \mathrm{l} /$ well. The cells were incubated for $24 \mathrm{~h}$. The cells were washed with PBS and then fixed with $4 \%$ paraformaldehyde for 30 min. After washing with PBS once again, the PBS containing $0.3 \%$ Triton X-100 was added and incubated for $5 \mathrm{~min}$ at room temperature. Then the cells were washed with PBS twice and $50 \mu$ l of TUNEL assay solution (prepared according to the instructions) was added and incubated at $37^{\circ} \mathrm{C}$ for $60 \mathrm{~min}$ in the dark. The cells were washed with PBS three times and sealed with the antifade mounting medium and observed under a fluorescence microscope (EX: 450-500 nm, EM: 515-565 nm).

Migration assay

The migration activity of BC cells was evaluated by wound healing assay. Briefly, after transfection, the cells were digested and collected, washed with PBS, and then resuspended in DMEM medium to $1 \times 10^{6}$ cells $/ \mathrm{ml}$. The cell suspensions were added to a 6 -well culture plate at $1 \mathrm{ml} /$ well. After the cells were covered with the bottom of the plate, the cell layers were scratched by a perpendicular tip to form a wounded gap, and the scraped cells were washed away with PBS. The serum-free medium was added and incubated for $24 \mathrm{~h}$. Finally, the gap width was measured. 
Invasion assay

The invasion activity of $\mathrm{BC}$ cells was determined by the Transwell chamber assay. First, the matrigel was diluted to $1 \mathrm{mg} / \mathrm{ml}$ and $100 \mu \mathrm{l}$ diluted matrigel was added to the upper chamber. Then, after transfection, the cells were digested and collected, washed with PBS, and then resuspended in serum-free DMEM to $1 \times 10^{4} \mathrm{cells} / \mathrm{ml}$. Then $400 \mu \mathrm{l}$ cell suspension was added to the upper chamber and $1 \mathrm{ml}$ DMEM containing $10 \%$ serum was added to the lower chamber. The cells in the chamber were incubated in an incubator for $24 \mathrm{~h}$, then the cells were fixed with methanol for $20 \mathrm{~min}$, and stained with crystal violet for $20 \mathrm{~min}$. The cells on the chamber membrane were wiped with a cotton swab and then observed and photographed under the microscope.

\section{QRT-PCR assay}

The total RNAs of BC tissues and cells were extracted with Trizol reagent (Invitrogen), the total miRNAs were extracted with miRcute miRNA isolation kit (Genconway) according to the manufacturer's instructions. For miRNA assay, the reverse transcription of the first strand of cDNA was performed by miRNA first-strand cDNA synthesis kit (TIANGEN). Briefly, the poly A tail was added to the 3 ' end of miR195-5p, and then the Poly $(A)$ modified miR-195-5p was subjected to reverse transcription to generate the first strand of cDNA according to the manufacturer's instructions. The qRT-PCR assay of the miR-195-5p cDNA was performed by miRcute miRNA fluorescence quantification kit (TIANGEN). Reaction system: 20 $\mu \mathrm{l}$, reaction conditions: $94^{\circ} \mathrm{C}$ for $2 \mathrm{~min},\left(94^{\circ} \mathrm{C}\right.$ for $20 \mathrm{~s}, 60^{\circ} \mathrm{C}$ for $30 \mathrm{~s}, 72^{\circ} \mathrm{C}$ for $\left.30 \mathrm{~s}\right) \times 45 \mathrm{cycle}$. U6 was used as an internal control. For mRNA assay, the reverse transcription of the first strand of cDNA was performed by iScript cDNA Synthesis kit (Bio-Rad) according to the manufacturer's instructions. Then the cDNAs were subjected to qRT-PCR assay with an SYBR Green Realtime PCR kit (TOYOBO). Reaction system: SYBR Green Realtime PCR Master Mix $7.5 \mu \mathrm{l}$, upstream and downstream primers each $0.25 \mu \mathrm{l}$, RT product $1 \mu \mathrm{l}$, and PCR water supplemented to $15 \mu \mathrm{l}$. Reaction conditions: $94^{\circ} \mathrm{C}$ for $2 \mathrm{~min},\left(94^{\circ} \mathrm{C}\right.$ for $15 \mathrm{~s}$, $55^{\circ} \mathrm{C}$ for $30 \mathrm{~s}, 72{ }^{\circ} \mathrm{C}$ for $\left.30 \mathrm{~s}\right) \times 45$ cycles. GAPDH was used as an internal control. The relative expression levels were calculated using $2^{-\triangle \Delta C T}$ method. The corresponding primers were in Table $S 1$ in the supplemental material.

Western blot

The BC tissues or cells were lysed with pre-cooled RIPA lysate (Sigma-Aldrich) and protease inhibitor cocktail (Beyotime). Then the tissue or cells were homogenized on ice, and the homogenates centrifuged at $12000 \mathrm{rpm}$ for $10 \mathrm{~min}$ at $4{ }^{\circ} \mathrm{C}$. The supernatants were collected and the protein concentration was measured by the BCA method. Then the loading buffer was added to the proteins and boiled for $10 \mathrm{~min}$ in a water bath. The proteins were subjected to SDS-PAGE gel electrophoresis at $20 \mu \mathrm{g} / \mathrm{well}$, and then the proteins were transferred to a PVDF membrane. The transferred PVDF membrane was washed in TBST for 5 min and blocked with $5 \%$ skim milk for $2 \mathrm{~h}$ at room temperature. Then the PVDF membrane was placed in a hybridization bag and probed with primary anti-VEGFA (\#ab1316, Abcam), anti-Ras (\#ab16907, Abcam), anti-phospho-Raf-1 (\#ab173539, Abcam), anti-Raf-1 (\#ab50858, Abcam), anti- 
phospho-ERK1/2 (\#ab214362, Abcam), ERK1/2 (\#ab54230, Abcam), anti-phospho-MEK1/2 (\#2338, Cell Signaling Technology), anti-MEK1/2 (\#4694, Cell Signaling Technology), and GAPDH (\#ab8245, Abcam) antibody that diluted with the blocking solution were added and incubated at $4{ }^{\circ} \mathrm{C}$ overnight. The PVDF membrane was washed with TBST buffer three times for 10 min each time, then the secondary antibody diluted in blocking solution (HRP goat anti-mouse) was added and incubated at $37^{\circ} \mathrm{C}$ for $1.5 \mathrm{~h}$. The immunoreactive protein bands were visualized by chemiluminescence using an Amersham prime ECL Plus detection system (GE Healthcare Life Sciences).

Subcellular fractionation assay

The cytoplasmic and nuclear were extracted from BC cells using the PARIS ${ }^{\text {TM }}$ Kit (Invitrogen). The extracts of cytoplasmic and nuclear were subjected to qRT-PCR assay. U6 as nuclear control, GAPDH as the cytoplasmic control.

\section{Dual-luciferase reporter assay}

The binding was determined by dual-luciferase reporter assay system (Promega). The target sequences were amplified by PCR and inserted into the pmirGLO vector (Promega) to construct the luciferase reporter vectors (pmirGLO-LINC00662-wt, pmirGLO-LINC00662-mut, pmirGLO-VEGFA-wt, pmirGLO-VEGFAmut). Then these reporter vectors were co-transfected with or without miR-195-5p mimics (NC mimics as a control) into HEK-293T cells, respectively. The luciferase activity was determined on an illuminometer (Berthold, Germany).

RNA pull-down assay

Firstly, the RNA was biotinylated with Biotin RNA Labeling Mix (Roche) and transcribed with T7 polymerase (Promega). Then the DNA was digested with RNase-free DNase I (Promega) and purified by RNeasy Mini Kit (QIAGEN). Three $\mu \mathrm{g}$ of biotinylated RNA was added to RNA structure buffer (10 mM Tris $\mathrm{pH} 7.0,0.1 \mathrm{M} \mathrm{KCl}, 10 \mathrm{mM} \mathrm{MgCl}$ ) and treated at $90^{\circ} \mathrm{C}$ for $2 \mathrm{~min}$, then placed on ice for $2 \mathrm{~min}$, and stood at room temperature for $30 \mathrm{~min}$. The T24 or RT 4 cells ( $1 \times 10^{7}$ cells) were suspended in $2 \mathrm{ml}$ of PBS, then 2 $\mathrm{ml}$ of RIPA lysis buffer was added, and the cells were lysed for $1 \mathrm{~h}$ at $4{ }^{\circ} \mathrm{C}$. The lysate was centrifuged at $12000 \mathrm{~g}$ for $10 \mathrm{~min}$ at $4^{\circ} \mathrm{C}$, and the supernatant was collected and transferred to an RNase-free tube. Then $400 \mathrm{ng}$ biotinylated RNA and $500 \mu \mathrm{l}$ RIP buffer were added to the tube and incubated for $1 \mathrm{~h}$, then $50 \mu$ of streptavidin agarose beads were added and incubated for $1 \mathrm{~h}$. The beads were washed with RIP buffer 5 times and washing solutions were collected for RT-PCR or western blot assay.

RIP assay

RIP assay was conducted in T24 and RT4 cells using Magna RIP RNA-binding protein immunoprecipitation kit (Millipore). Briefly, the cells were collected after washing with cold PBS and the RIP lysis buffer was added. Then the suspension was centrifugated and $100 \mu$ cell lysates were transferred to the RIP immunoprecipitation buffer which contained Ago2-conjugated magnetic beads, the 
mouse IgG as a negative control (Millipore, MA, USA). The magnetic beads washed with RIP wash buffer and then incubated with proteinase $\mathrm{K}$ for $30 \mathrm{~min}$ at $55^{\circ} \mathrm{C}$. The RNA was extracted for qRT-PCR assay.

Fluorescence in situ hybridization (FISH) assay

The presence of LINC00662 was determined by using a FISH kit (Boye Biological) and fluorescein isothiocyanate (FITC)-conjugated LINC00662 DNA probe (5'-CCAGGAGGCAGACCTTGT-3'). Briefly, the cells were seeded in a 24-well culture plate placing a slide. After the cells were covered, the cells were washed with cold PBS twice and fixed for 15 min with $4 \%$ RNase-free PFA. Then the cells were washed with PBS and incubated with $0.5 \%$ Triton X100 for 5 min, and then treated with gradient ethanol (80\%-90\%-100\%). Then hybridized with the probe and acquired image under a confocal laser microscope (Leica, Solms, German).

Tumor growth in vivo

Balb/c mice were depilated on the back, and then $100 \mu$ l of T24 cells successfully transfected with shLINC00662 were subcutaneously injected into the left dorsal ( $1 \times 10^{7}$ cells/mouse). The tumor volumes were monitored weekly for 5 weeks. On day 35 , the mice were sacrificed and the tumors were excised and weighted.

IHC staining

First, the sections were dewaxed and hydrated, then stained by SABC method, and the antigen was repaired by heat and blocked by $5 \%$ BSA blocking solution. Then, the sections were incubated with Ki-67 (\#ab156956, Abcam) or LINC00662 probe (5'-CCAGGAGGCAGACCTTGT-3') primary antibody overnight at $4{ }^{\circ} \mathrm{C}$ and incubated with secondary goat anti-rabbit IgG antibody (\#ab6721, Abcam) and horseradishstreptavidin working solution for $1.5 \mathrm{~h}$. Then the sections were subjected to DAB staining and hematoxylin counterstaining, dehydration, xylene transparent, neutral gum seal. Images were taken with a microscope.

Statistical analysis

All data were expressed as means \pm SD. Student's t test, One-way ANOVA, Kaplan-Meier's method, and the log-rank test were performed by SPSS 13.0. All data from at least three independent experiments. Statistical significance was accepted at $P$ values $<0.05$.

\section{Results}

LINC00662 is up-regulated in BC tissues and cells

We first examined the expression of LINC00662 in BC tissues and adjacent normal tissue samples from 60 BC patients by qRT-PCR. As shown in Fig. 1A, the expression level of LINC00662 in BC tissues was significantly higher than that in normal tissues (about 2 times) $(n=60, P<0.0001)$. Then, these BC 
patients were divided into high LINC00662 group ( $\geq$ median, $\mathrm{n}=27$ ) and low LINC00662 group (<median, $n=33$ ) according to the expression level of LINC00662. The 5-year survival of these patients was recorded. As shown in Fig. 1B, the low LINC00662 expression patients showed a better survival rate compared with that of high LINC00662 expression ( $\mathrm{P}=0.0105)$. In addition, we also evaluated the expression of LINC00662 in a human bladder epithelium immortalized cells (SV-HUC-1) and several BC cell lines (T24, RT4, 5637, J82, and SW780). The results suggested that the LINC00662 was up-regulated in the BC cell lines (T24, RT4, 5637, J82, and SW780) than that of SV-HUC-1 cells (more than 4-fold, Fig. 1C). The IHC assay also demonstrated that LINC00662 was highly expressed in BC tissues (Fig. 1D).

LINC00662 promotes BC cell proliferation in vitro and growth in vivo

In order to investigate the possible role of LINC00662 in BC, the expression vector carried LINC00662 or shRNA against LINC00662 was constructed and transfected into the T24 and RT4 cells to over-express or knockdown the LINC00662 expression. As shown in Fig. 2A, pcDNA3.1-LINC00662 and sh-LINC00662 successfully up-regulated and down-regulated the expression of LINC00662 in T24 and RT4 cells, respectively. Subsequently, the effects of pcDNA3.1-LINC00662 and sh-LINC00662 on BC cells proliferation and apoptosis were examined. As shown in Fig. 2B, pcDNA3.1-LINC00662 obviously promoted the proliferation of T24 and RT 4 cells, however, this promotion effect was inhibited by shLINC00662 significantly. The apoptosis effect of LINC00662 on the BC cell was detected by TUNEL staining assay. The results suggested that sh-LINC00662 increased the number of apoptotic cells, whereas pcDNA3.1-LINC00662 remarkably inhibited BC cells apoptosis (Fig. 2C-D). Moreover, the effect of LINC00662 on BC growth was also investigated by a subcutaneous xenograft mouse model. We found that sh-LINC00662 drastically inhibited tumorigenicity of BC cells, and showed a smaller tumor volume and weight compared with the control group mice (Fig. 2E). The sh-LINC00662 group mice also presented a lower LINC00662 and Ki67 expression compared with the control group which determined by QRT-PCR and IHC (Fig. 2F).

LINC00662 increases the migration and invasion activities BC cells

Our study has demonstrated that LINC00662 over-expression promoted the proliferation activity of BC cells in vitro. In this part, we investigated the effect of LINC00662 on BC cell migration and invasion activity. As shown in Fig. 3A, both the control group of pcDNA3.1-NC and sh-NC showed a similar relative wound gap width, while pcDNA3.1-LINC00662 accelerated the healing of the wound gap and shLINC00662 inhibited the wound healing. Furthermore, pcDNA3.1-LINC00662 significantly promoted the ability of BC cells to invade through Matrigel, while sh-LINC00662 significantly reduced this invasion behavior (Fig. 3B). Therefore, these results suggested that LINC00662 has a role in promoting BC cells migration and invasion.

\section{LINC00662 targets miR-195-5p}

The expression and location of LINC00662 in BC cells were detected by FISH and subcellular fractionation assay. The results suggested that the LINC00662 mainly expressed in the cytoplasm (Fig. 
4A-B). We predicted the possible target miRNAs for LINC00662 via Starbase. Through bioinformatics prediction, we found that LINC00662 may target miR-195-5p. The complementary binding sequence with miR-195-5p and a mutant sequence of LINC00662 weres shown in Fig. 4C. We performed a dualluciferase reporter assay to provide further validation. It was found that miR-195-5p mimics significantly reduced the luciferase activity of LINC00662-wt, while had no significant effect on LINC00662-mut, indicating that miR-195-5p can bind to LINC00662 sequence (Fig.4D). In order to verify whether LINC00662 and miR-195-5p bind through the ribonucleoprotein complex of miRNA, RIP assay and Ago2 antibody were used to capture the Ago2 protein and its binding RNA. The results showed that LINC00662 and miR-195-5p were combined with Ago2 (Fig. 4E). In addition, their binding was also validated by biotin-labeled RNA pull-down assay. As shown in Fig.4F, a large amount of Bio-miR-195-5p WT was detected in the LINC00662 pull-down pellets compared to the Bio-NC control or the Bio-miR-195-5p MUT group by qRT-PCR.

In addition, our results also suggested that over-expression of LINC00662 significantly decreased miR195-5p level and knockdown of LINC00662 displayed an opposite effect in BC cells (Fig. 4G). So the expression of miR-195-5p in the BC tissues and its relationship with LINC00662 were further studied. The results showed that the expression level of miR-195-5p in BC tissues was significantly lower than that in normal tissues, which was completely opposite to the expression of LINC00662 (Fig. 4H). These results suggested that LINC00662 may directly bind to miR-195-5p to inhibit its activity.

MiR-195-5p targets VEGFA and inhibits BC cells proliferation, migration, and invasion

The downstream targets for miR-195-5p were also predicted by bioinformatics (TargetScan). According to the sequence match, VEGFA may be the target of miR-195-5p (Fig. 5A). Their binding was determined by dual-luciferase reporter assay. As shown in Fig. 5B, miR-195-5p mimics significantly reduced the luciferase activity of LINC00662-wt, while had no significant effect on LINC00662-mut, indicating that miR-195-5p can bind to VEGFA sequence directly. Furthermore, the effect of miR-195-5p on the VEGFA expression was studied. The expression efficiencies of the inhibitor and mimics of miR-195-5p were confirmed in Fig. 5C. The results showed that the mRNA and protein expression levels of VEGFA in BC cells were significantly decreased by miR-195-5p mimics and significantly increased by miR-195-5p inhibitor (Fig. 5D-E). However, pcDNA3.1-VEGFA could abrogate the regulation of miR-195-5p mimics on the VEGFA expression in BC cells (Fig. 5F). The expression of VEGFA in the BC tissues and its relationship with miR-195-5p were further investigated. The results showed that the expression level of VEGFA in BC tissues was significantly higher than that in normal tissues, which was negatively correlated with the miR195-5p expression (Fig. 5G). Moreover, the role of miR-195-5p and VEGFA in the proliferation, apoptosis, migration, and invasion of $B C$ cells were evaluated. The results showed that up-regulation of miR-195-5p showed anti-tumor effects such as inhibition of proliferation, induction of apoptosis, and inhibition of invasion and migration, while these effects were abrogated when the pcDNA3.1-VEGFA was cotransfection simultaneously (Fig. 5H-K). These results suggested that miR-195-5p may inhibit BC cells proliferation and invasion through targeting VEGFA. 
LINC00662 regulates BC cells proliferation and invasion activity via miR-195-5p-mediated VEGFA/Ras/Raf/MEK/ERK signaling pathway.

The underlying molecular mechanisms of LINC00662 and miR-195-5p on BC were further explored. SiLINC00662 and miR-195-5p inhibitor and their corresponding controls were transfected into BC cells and their effects on the expression of LINC00662, miR-195-5p, and VEGFA were evaluated. As shown in Fig. 6A-C, si-LINC00662 significantly increased miR-195-5p expression and decreased VEGFA expression, while miR-195-5p inhibitors increased the VEGFA expression in si-LINC00662-treated cells. In addition, knockdown of LINC00662 inhibited the proliferation, migration, and invasion activity and increased the apoptosis of BC cells (Fig. 6D-G and Fig. 7A-B). However, these regulatory effects of si-LINC00662 on BC cells were effectively reversed by miR-195-5p inhibitors. Furthermore, we evaluated the regulation of LINC00662 on VEGFA and Ras/ERK signaling pathway. As shown in Fig. 7C, knockdown of LINC00662 reduced VEGFA expression significantly but had no significant effect on Ras expression. Knockdown of LINC00662 had no effect on the expression of Raf-1, MEK1/2, and ERK1/2 but significantly inhibited their phosphorylation. However, these regulatory effects of si-LINC00662 were abrogated by miR-195-5p inhibitor. These results indicated that LINC00662 regulated BC cells proliferation and invasion activity probably via miR-195-5p-mediated VEGFA/ Ras/Raf/MEK/ERK signaling pathway.

\section{Discussion}

LncRNA is a non-coding RNA that consists of more than 200 nucleotides, which is widely distributed in the genome. LncRNA has been considered as a "garbage" in transcription activity, but in fact, they have multiple biological functions and participate in most physiological and pathological processes in the body ${ }^{14}$. The discovery of non-coding RNA regulatory functions has overturned the understanding of gene expression regulation. To date, more than 10000 IncRNAs have been found in human genes. Studies have found that IncRNA plays an important role in the development and progression of various tumors through chromatin modification, RNA splicing, and protein regulation. The abnormal expression of IncRNAs can promote or inhibit the expression of some oncogenes. In recent years, the important biological functions of IncRNAs in the development and progression of tumors and its potential value in screening biomarkers for early diagnosis or prognosis of tumors have attracted much attention ${ }^{15,16}$.

$\mathrm{BC}$ is a urological malignancy that has particularly high morbidity worldwide ${ }^{17}$. Patients with $\mathrm{BC}$ tend to have a high rate of recurrence and a bad survival rate. In recent decades, the scientist has made a lot of efforts to improve early diagnosis and treatment approaches, however, the reliable prognostic markers and effective chemotherapeutics for $\mathrm{BC}$ are still unsatisfactory and dearly needed ${ }^{18,19}$. The tumorigenesis and progression in $\mathrm{BC}$ are complex processes involving multiple oncogenes activation and tumor suppressor genes inactivation, and recent studies have shown that IncRNA plays an important role in these processes ${ }^{20,21}$. Some IncRNAs were identified to express in tumor and normal tissues differentially and considered to have potential as a marker for tumor diagnosis and prognosis ${ }^{22,23}$. In this study, we found that LINC00662 is not only up-regulated in BC tissues, but also highly expressed in 
various BC cell lines such as T24, RT4, 5637, J82, and SW780 cells, however, it shows a lower level in normal tissues and human bladder epithelium cells. We also collected the 5-year survival data of enrolled BC patients and analyzed its relationship with LINC00662 expression by Kaplan-Meier's method. The statistical assay showed that the expression of LINC00662 in BC tissues was negatively correlated with the 5-year survival rate of the patients.

In order to illustrate the biological role of LINC00662 in the development of BC more comprehensively, we achieved the regulation of LINC00662 expression by transfecting over-expressing or silencing plasmids to investigate its effect on the biological behavior of $B C$ cells. Our results showed that over-expression of LINC00662 promoted the proliferative activity and inhibited the apoptosis of BC cells, showing a protumor effect, while knockdown of LINC00662 showed an opposite effect. In addition, a subcutaneous xenograft mouse model was established to assess the effect of LINC00662 on tumor growth in vivo. Consistent with the in vitro results, knockdown of LINC00662 inhibited BC growth in mice. Furthermore, the migration and invasion were also investigated and the results suggested that LINC00662 promoted migration and invasion of BC cells. These data indicated that LINC00662 could regulate BC malignant cell phenotype in vivo and in vitro.

MiRNA is a class of endogenous non-coding small RNAs containing 17-25 nucleotides that play an important role in the regulation of various biological processes such as cell growth, division, metabolism and development ${ }^{24}$. In recent decades, the biological function and role of miRNA in tumors were explored intensively and extensively. Numerous evidence has confirmed that the abnormal expression of miRNA is closely related to the tumorigenesis, development, and metastasis of various tumors ${ }^{25}$. Many studies have shown that miRNA is an important link in the role of IncRNA. LncRNA can absorb specific miRNA by sponge effect or competitively bind miRNA to regulate the binding of endogenous miRNA to its target gene ${ }^{26}$. In this study, we predicted and validated the possible interact target of LINC00662. We found that the downstream target is miR-195-5p, which was reported to act as a tumor suppressor and could regulate the proliferation and invasion of various tumor cells such as $\mathrm{BC}{ }^{27}$, adrenocortical carcinoma ${ }^{28}$ anf hepatocellular carcinoma ${ }^{29}$. Fei et al. ${ }^{27}$ reported that miR-195-5p suppressed glucose uptake and proliferation of human $\mathrm{BC}$ cell line $\mathrm{T} 24$, however, its underlying regulation effect on $\mathrm{BC}$ remains to be further investigated. It is noteworthy that, contrary to the expression of LINC00662, miR-195-5p was down-regulated in BC tissues, and the in vitro results suggested that LINC00662 negatively regulated the miR-195-5p expression. From these findings, we believed that LINC00662 might act as an endogenous sponge RNA to interact with miR-195-5p in BC.

We further predicted and justified that VEGFA, is the target for miR-195-5p ${ }^{30}$. VEGFA is considered to be one of the important drivers of cancer angiogenesis and reported to related with increasing of vascular distribution, metastasis and leading to the poor prognosis of various cancers ${ }^{30}$. Studies have shown that the Ras/Raf/MEK/ERK signaling cascade plays a key role in the VEGF-mediated angiogenic signaling pathway for signaling and promoting VEGF expression ${ }^{31,32}$. Our present study showed that knockdown of LINC00662 up-regulated the miR-195-5p expression and then down-regulated the VEGFA expression, 
ultimately showed proliferation, migration, and invasion inhibitory activity and pro-apoptotic activity. Moreover, the decrease of VEGFA expression caused by LINC00662 knockdown inhibited Raf-1, MEK1/2, and ERK1/2 phosphorylation, while the miR-195-5p inhibitor counteracted this regulation effect. These results indicated that LINC00662 regulated the proliferation, apoptosis, migration, and invasion of BC cells probably via miR-195-5p mediated VEGFA/Ras/Raf/MEK/ERK signaling pathway.

\section{Conclusion}

In summary, our study demonstrated that LINC00662 is up-regulated in BC tissues and cell lines. Knockdown of LINC00662 showed a tumor inhibition effect, which was antagonized by miR-195-5p or VEGFA. These findings suggested that the LINC00662 plays a regulatory role in the development and invasion of BC through miR-195-5p-mediated VEGFA/Ras/Raf/MEK/ERK signaling pathway.

\section{Abbreviations}

\begin{tabular}{ll} 
BC & Bladder cancer \\
\hline LncRNA & LncRNA Long non-coding RNA \\
\hline QRT-PCR & Quantitative real-time polymerase chain reaction \\
\hline ISH & In situ hybridization \\
\hline miRNA & microRNA \\
\hline FISH & Fluorescence in situ hybridization
\end{tabular}

\section{Declarations}

\section{Ethics approval and consent to participate}

The present study was approved by the Institutional Ethical Review Committee of The Affiliated Huaian No.1 People's Hospital of Nanjing Medical University.

\section{Consent for publication}

Not applicable.

\section{Availability of data and material}

The data used in this study is available on a reasonable request from the corresponding author.

\section{Competing interests}

The authors declare that they have no competing interests. 


\section{Funding}

Not applicable.

\section{Authors' contributions}

All listed authors designed the study, performed the experiments and the statistical analysis, and wrote the manuscript. All authors read and approved the final manuscript.

\section{Acknowledgements}

We thank the Department of Pathology of The Affiliated Huaian No.1 People's Hospital of Nanjing Medical University.

\section{Authors' information}

Not applicable.

\section{References}

1. Siegel RL., Miller KD. \& Jemal A. Cancer statistics. Ca A Cancer Journal for Clinicians. 67(1): 7-30 (2017).

2. Chen Wg, et al. Cancer statistics in China. Ca Cancer J Clin. 66(2): 115-132 (2015).

3. Sanli O, Dobruch J, Knowles MA., Burger M \& Lotan Y. Bladder Cancer. Nature Reviews Disease Primers. 3: 17022 (2017).

4. Mercer TR., Dinger ME. \& Mattick JS. Long non-coding RNAs: insights into functions. Nature Reviews Genetics, 10(3): 155-159 (2009).

5. Feng $\mathrm{J}$, et al. LncRNA PCNAP1 modulates hepatitis B virus replication and enhances tumor growth of liver cancer. Theranostics. 9(18): 5227-5245 (2019).

6. Zhang $S$, et al. Evaluation of serum exosomal LncRNA-based biomarker panel for diagnosis and recurrence prediction of bladder cancer. Journal of cellular and molecular medicine. 23(2): 1396- 
1405 (2019).

7. Yu C, et al. LncRNA PVT1 regulates VEGFC through inhibiting miR-128 in bladder cancer cells. Journal of cellular physiology. 234(2): 1346-1353 (2019).

8. Zhong X, Long Z, Wu S, Xiao M \& Hu W. LncRNA-SNHG7 regulates proliferation, apoptosis and invasion of bladder cancer cells assurance guidelines. Journal of BUON : official journal of the Balkan Union of Oncology. 23(3): 776-781 (2018).

9. Fan Y, et al. Long non-coding RNA UCA1 increases chemoresistance of bladder cancer cells by regulating Wnt signaling. The FEBS journal. 281(7): 1750-1758 (2014).

10. Xie $\mathrm{H}$, et al. LncRNA MALAT1 Inhibits Apoptosis and Promotes Invasion by Antagonizing miR-125b in Bladder Cancer Cells. Journal of Cancer. 8(18): 3803-3811 (2017).

11. Gong W, Su Y, Liu Y, Sun P \& Wang X. Long non-coding RNA Linc00662 promotes cell invasion and contributes to cancer stem cell-like phenotypes in lung cancer cells. Journal of biochemistry. 164(6): 461-469 (2018).

12. Liu Z, et al. LINC00662 promotes gastric cancer cell growth by modulating the Hippo-YAP1 pathway. Biochemical and biophysical research communications. 505(3): 843-849 (2018).

13. Xu D, Chen Y, Yuan C, Zhang S \& Peng W. Long non-coding RNA LINC00662 promotes proliferation and migration in oral squamous cell carcinoma. OncoTargets and therapy. 12(undefined): 647-656 (2019).

14. Sahu A, Singhal U \& Chinnaiyan AM. Long Noncoding RNAs in Cancer: From Function to Translation. Trends Cancer. Oct 1;1(2):93-109 (2015). 
15. Wang $\mathrm{M}$, et al. Common and differentially expressed long noncoding RNAs for the characterization of high and low grade bladder cancer. Gene, 592(1): 78-85 (2016).

16. Zhu W, Liu H, Wang X, Lu J \& Yang W. Long noncoding RNAs in bladder cancer prognosis: A metaanalysis. Pathology, research and practice. 215(6): 152429 (2019).

17. Terracciano D, et al. Urinary long noncoding RNAs in nonmuscle-invasive bladder cancer: new architects in cancer prognostic biomarkers. Translational research : the journal of laboratory and clinical medicine. 184(undefined): 108-117 (2017).

18. Hyeon KJ, Kim W-J, P. Seth L, Chun F \& Luis AK. Diagnostic and Prognostic Markers in Bladder Cancer. Disease Markers. 2016: 1-2 (2016).

19. Fouad H, Salem H, Ellakwa DE \& Hamid MA. MMP-2 and MMP-9 as prognostic markers for the early detection of urinary bladder cancer. Journal of biochemical and molecular toxicology. 33(4): e22275 (2019).

20. Qiu F, Zhang MR, Zhou Z, Pu JX \& Zhao XJ. IncRNA MIR503HG functioned as a tumor suppressor and inhibited cell proliferation, metastasis and epithelial-mesenchymal transition in bladder cancer. Journal of cellular biochemistry. 120(6): 10821-10829 (2019).

21. Yu H, Wang S, Zhu H \& Rao D. LncRNA MT1JP functions as a tumor suppressor via regulating miR214-3p expression in bladder cancer. Journal of Cellular Physiology. doi: 10.1002 (2019).

22. Li Z, Hong S \& Liu Z. LncRNA LINC00641 predicts prognosis and inhibits bladder cancer progression through miR-197-3p/KLF10/PTEN/PI3K/AKT cascade. Biochemical and biophysical research communications. 503(3): 1825-1829 (2018). 
23. Tuo Z, Zhang J \& Xue W. LncRNA TP73-AS1 predicts the prognosis of bladder cancer patients and functions as a suppressor for bladder cancer by EMT pathway. Biochemical and biophysical research communications. 499(4): 875-881 (2018).

24. Nekoohesh L, Modarressi MH, Mowla SJ, Sadroddiny E, Etemadian M, Afsharpad M, et al. Expression profile of miRNAs in urine samples of bladder cancer patients. Biomarkers in medicine. 12(12): 13111321 (2018).

25. Amuran GG, Eyuboglu IP, Tinay I \& Akkiprik M. New Insights in Bladder Cancer Diagnosis: Urinary miRNAs and Proteins. Medical sciences (Basel, Switzerland). 6(4): undefined (2018).

26. Luo H, Xu C, Le W, Ge B \& Wang T. IncRNA CASC11 promotes cancer cell proliferation in bladder cancer through miRNA-150. Journal of cellular biochemistry. 120(8): 13487-13493 (2019).

27. Fei X, Qi M, Wu B, Song Y, Wang Y \& Li T. MicroRNA-195-5p suppresses glucose uptake and proliferation of human bladder cancer T24 cells by regulating GLUT3 expression. FEBS letters. 586(4): 392-397 (2012).

28. Soon PS, et al. miR-195 and miR-483-5p Identified as Predictors of Poor Prognosis in Adrenocortical Cancer. Clinical cancer research : an official journal of the American Association for Cancer Research. 15(24): 7684-7692 (2009).

29. Xu T, Zhu Y, Xiong Y, Ge YY, Yun JP \& Zhuang SM. MicroRNA-195 suppresses tumorigenicity and regulates G1/S transition of human hepatocellular carcinoma cells. Hepatology (Baltimore, Md). 50(1): 113-121 (2019).

30. Cao Y, et al. VEGF exerts an angiogenesis-independent function in cancer cells to promote their malignant progression. Cancer research. 72(16): 3912-3918 (2012). 
31. Yang H, Wan Z, Huang C, Yin H \& Song D. AMPH-1 is a tumor suppressor of lung cancer by inhibiting Ras-Raf-MEK-ERK signal pathway. Lasers in medical science. 34(3): 473-478 (2019).

32. Raza A, Pandey MS, Jin Q \& Mulder KM. km23-1/DYNLRB1 regulation of MEK/ERK signaling and RRas in invasive human colorectal cancer cells. Cell biology international. doi: 10.1002 (2019).

\section{Figures}

A
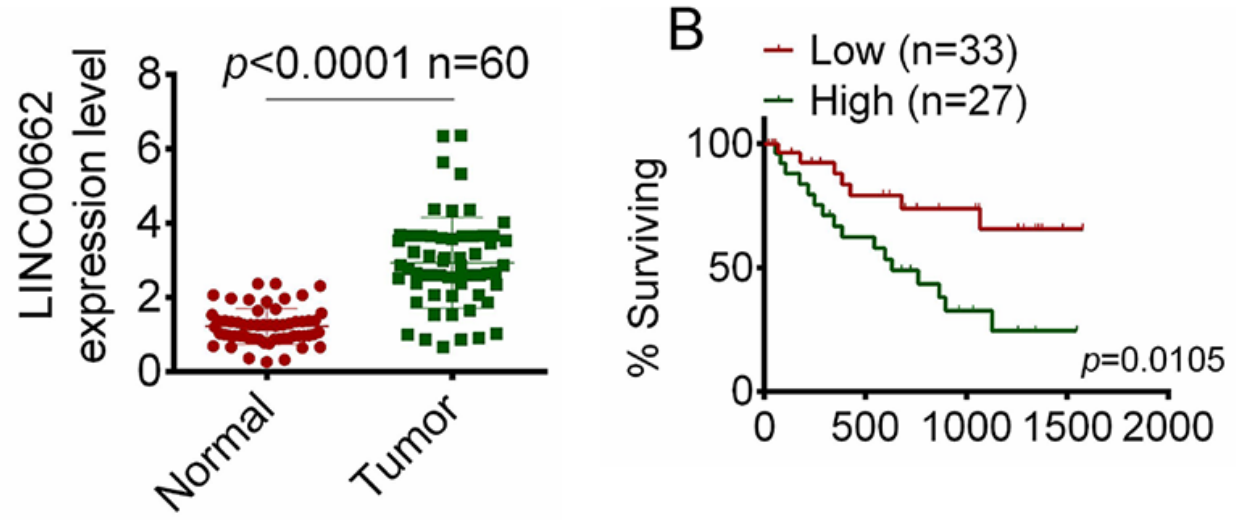

C

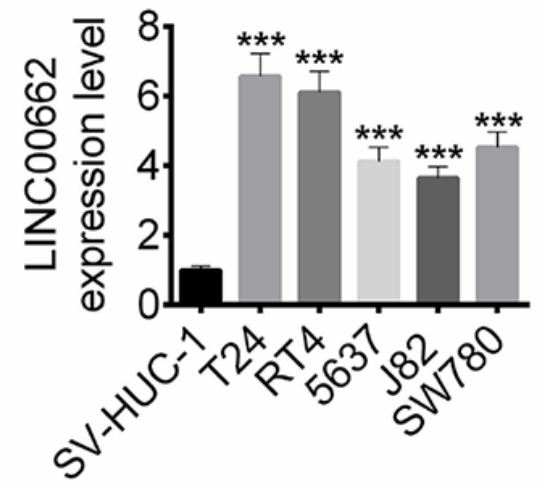

$\mathrm{D}$
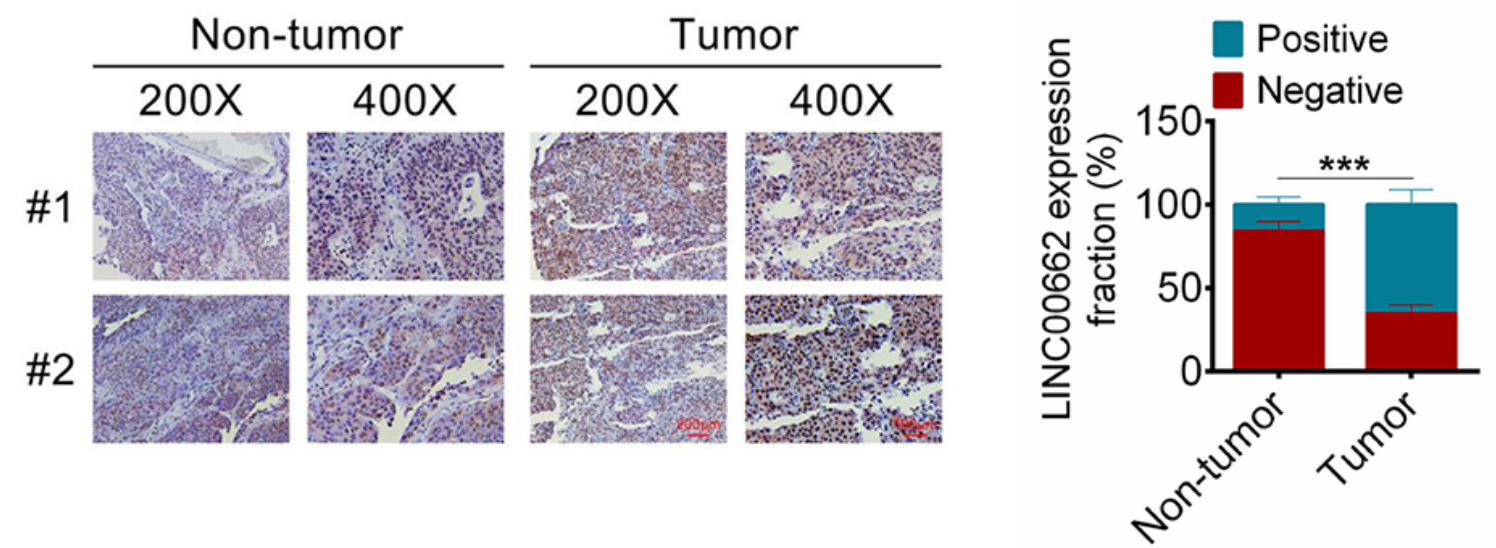

Figure 1

LINC00662 was up-regulated in BC tissues and cell lines. (A) The LINC00662 expression in 60 cases BC tissues and adjacent normal tissues were determined by qRT-PCR. LINC00662 was highly expressed in BC tissues. (B) The relationship of 5 year survival rate of the BC patients with high $(n=27) /$ low $(n=33)$ LINC00662 expression were analyzed by Kaplan-Meier's method and the log-rank test. The patients with a low LINC00662 expression level were presented a better survival rate than that of high LINC00662 expression level. (C) The mRNA expression level of LINC00662 in a human bladder epithelium immortalized cell line (SV-HUC-1) and five BC cell lines (T24, RT4, 5637, J82, and SW780) was determined by qRT-PCR. The LINC00662 was highly expressed in BC cells. (D) The expression of LINC00662 in BC 
tissues and normal tissues was determined by IHC assay. Data were expressed as the mean $\pm S D, n=3$. *P $<0.05, * * P<0.01, * * * P<0.001$ vs SV-HUC-1.

A
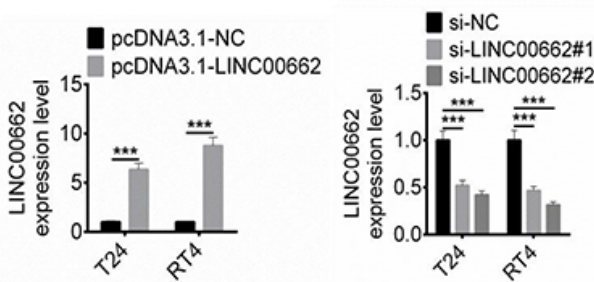

C

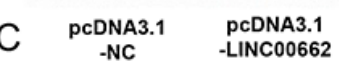

-NC -LINC00662
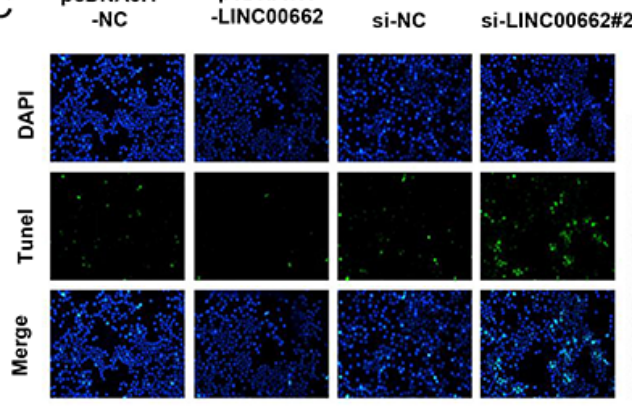

E

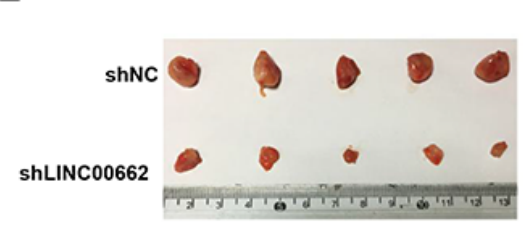

B
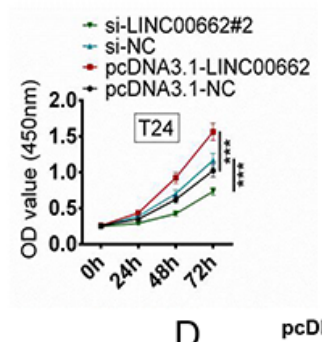

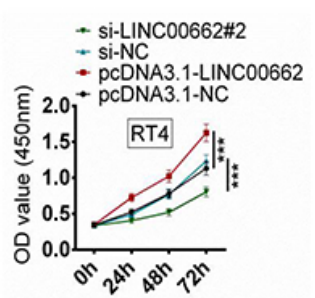

PcDNA3.1

-LINC00662 si-NC Si-LINC00662\#2

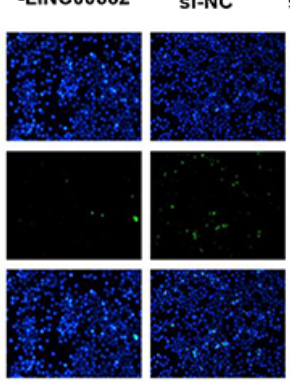

$\mathrm{F}$

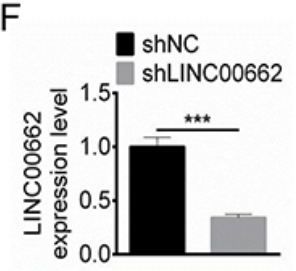

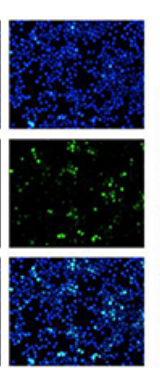
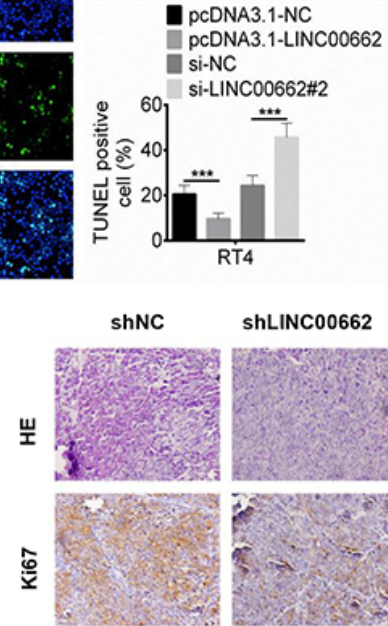

ShLINC00662

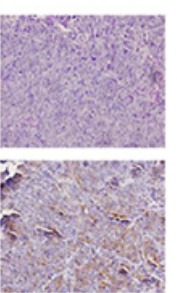

Figure 2

LINC00662 regulates proliferation and inhibits apoptosis of BC cells and tumor growth of BC mice. (A) The expression efficiency of LINC00662. The expression of LINC00662 was over-expressed by pcDNA3.1LINC00662 and knockdown by sh-LINC00662. (B) The proliferation of BC cells was determined by the CCK-8 assay. The proliferation of T24 and RT4 cells was promoted by over-expression of LINC00662 and inhibited by knockdown of LINC00662. (C) The apoptosis of BC cells was determined by TUNEL staining assay. The apoptosis of T24 and RT4 cells was inhibited by over-expression of LINC00662 and promoted by knockdown of LINC00662. (D) A subcutaneous BC tumor model was established to evaluate the antitumor effect of shLINC00662. Knockdown of LINC00662 inhibits the growth of the tumor (volumes and weights), tumor volume was caculated as follows the formula: Tumor volume $(\mathrm{mm} 3)=0.5 \times \mathrm{L} \times \mathrm{W} 2$, the $\mathrm{L}$ is the length and $W$ is the width. (E) The mRNA expression of LINC00662 in the BC mice tumor tissues was determined by qRT-PCR. (F) The Ki67 expression was determined by IHC assay. Data were expressed as the mean $\pm S D, n=3$. ${ }^{*} P<0.05, * \star P<0.01, * \star * P<0.001$. 


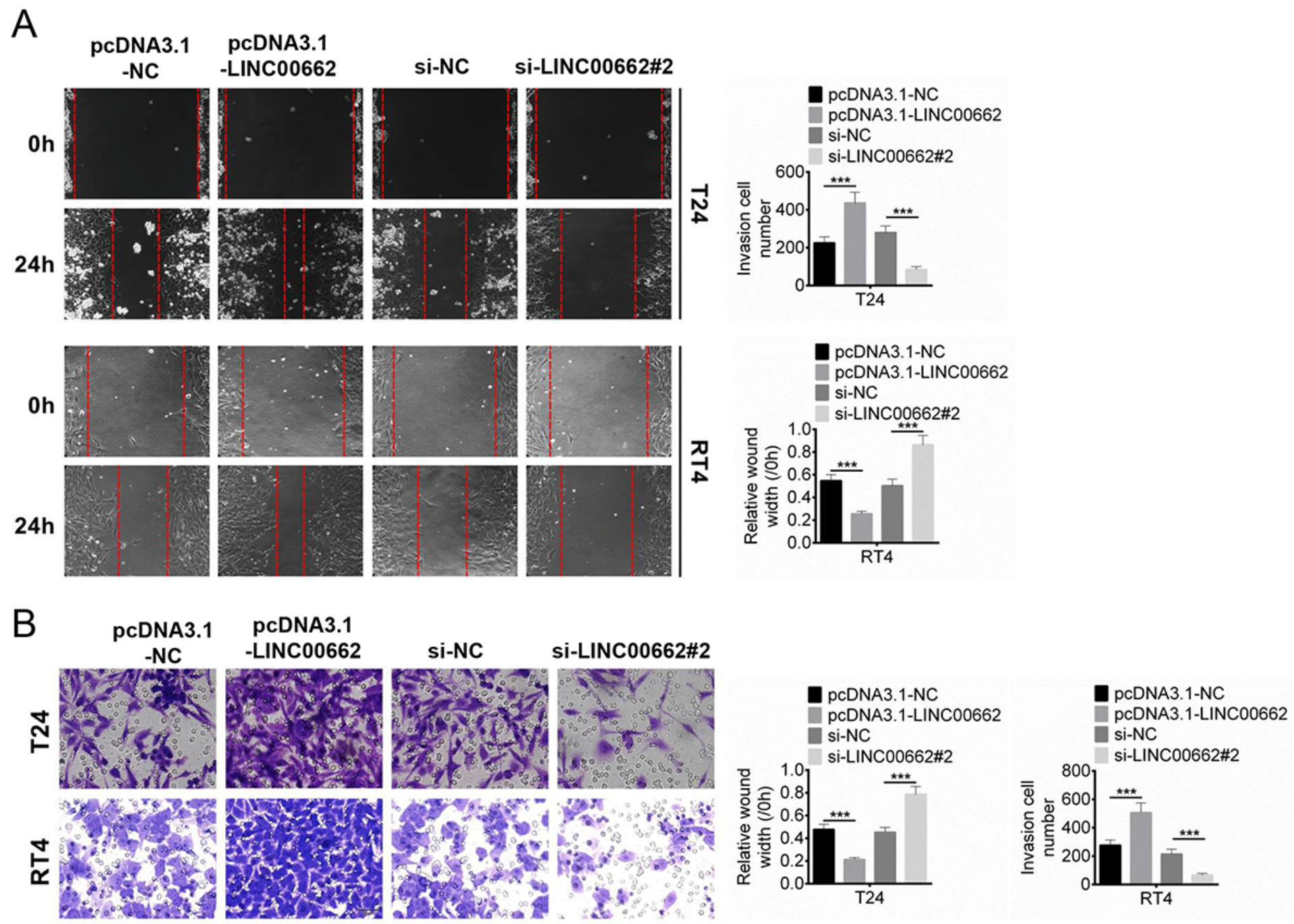

\section{Figure 3}

LINC00662 promotes migration and invasion of BC cells. (A) The migration of BC cells was determined by wound healing assay. Over-expression of LINC00662 accelerated the wound area closure, while retarded by knockdown of LINC00662. (B) The invasion of BC cells was determined by Transwell chamber assay. Over-expression of LINC00662 promoted the invasive ability of BC cells while reduced by knockdown of LINC00662. Data were expressed as the mean $\pm S D, n=3$. ${ }^{*} P<0.05, * * P<0.01$, ${ }^{\star \star *} P<$ 0.001 . 
A

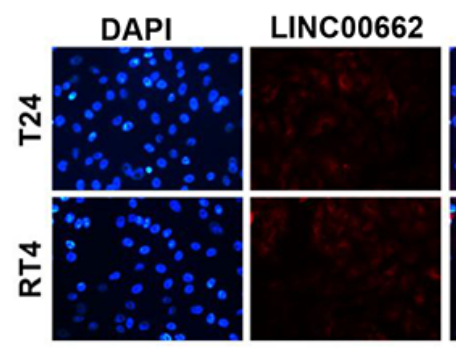

C

LINC00662 WT 5' UCAAACAAAAAGAUGCUGCUA 3' miR-195-5p 3' CGgUUAUAAAGACACGACGAU 5' LINC00662 MUT 5' UCAAACAAAAAGAUGCUGCUA 3'

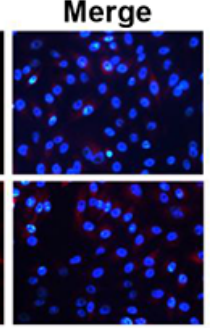

B
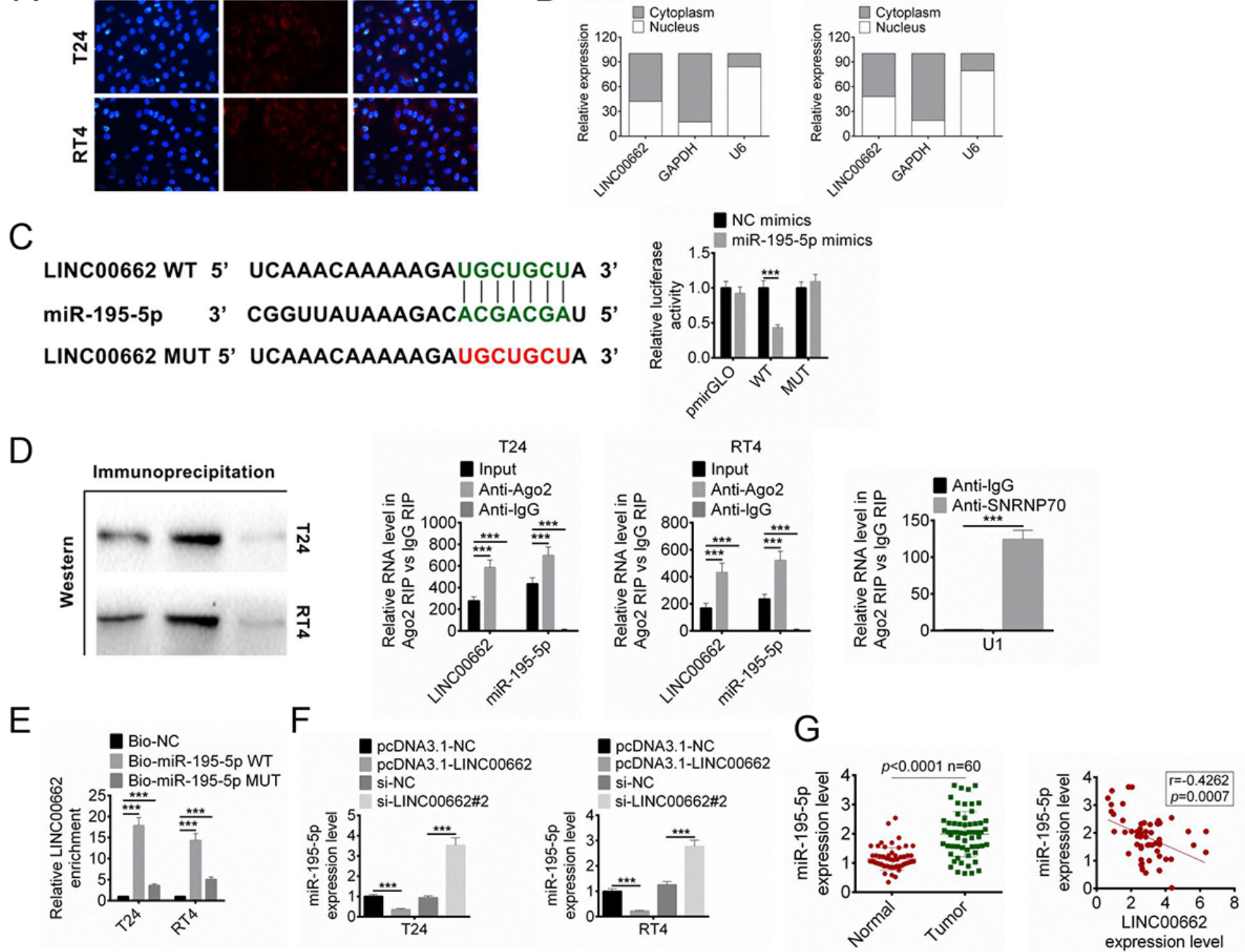

Figure 4

LINC00662 targets miR-195-5p. (A) The expression of LINC00662 in the BC cells was determined by FISH assay. (B) The specific location of LINC00662 was identified in BC cells through performing subcellular fractionation assay. LINC00662 mainly expressed in the cytoplasm. (C) The possible target miRNAs for LINC00662 was predicted by Starbase. (D) The binding of miR-195-5p and LINC00662 was verified by dual-luciferase reporter assay. (E) The binding of miR-195-5p and LINC00662 was validated by RIP assay. (F) The binding of miR-195-5p and LINC00662 was validated by RNA pull down assay. (G) The expression of miR-195-5p regulated by LINC00662 was determined by qRT-PCR. Over-expression of LINC00662 decreased miR-195-5p expression and knockdown of LINC00662 increased miR-195-5p expression. $(H)$ The expression of miR-195-5p in BC tissues and the relationship between LINC00662 and miR-195-5p expression. MiR-195-5p was down-regulated in BC tissues and negatively correlated with LINC00662 expression. Data were expressed as the mean $\pm S D, n=3 .{ }^{*} P<0.05,{ }^{\star *} P<0.01$, ${ }^{\star \star *} P<0.001$. 


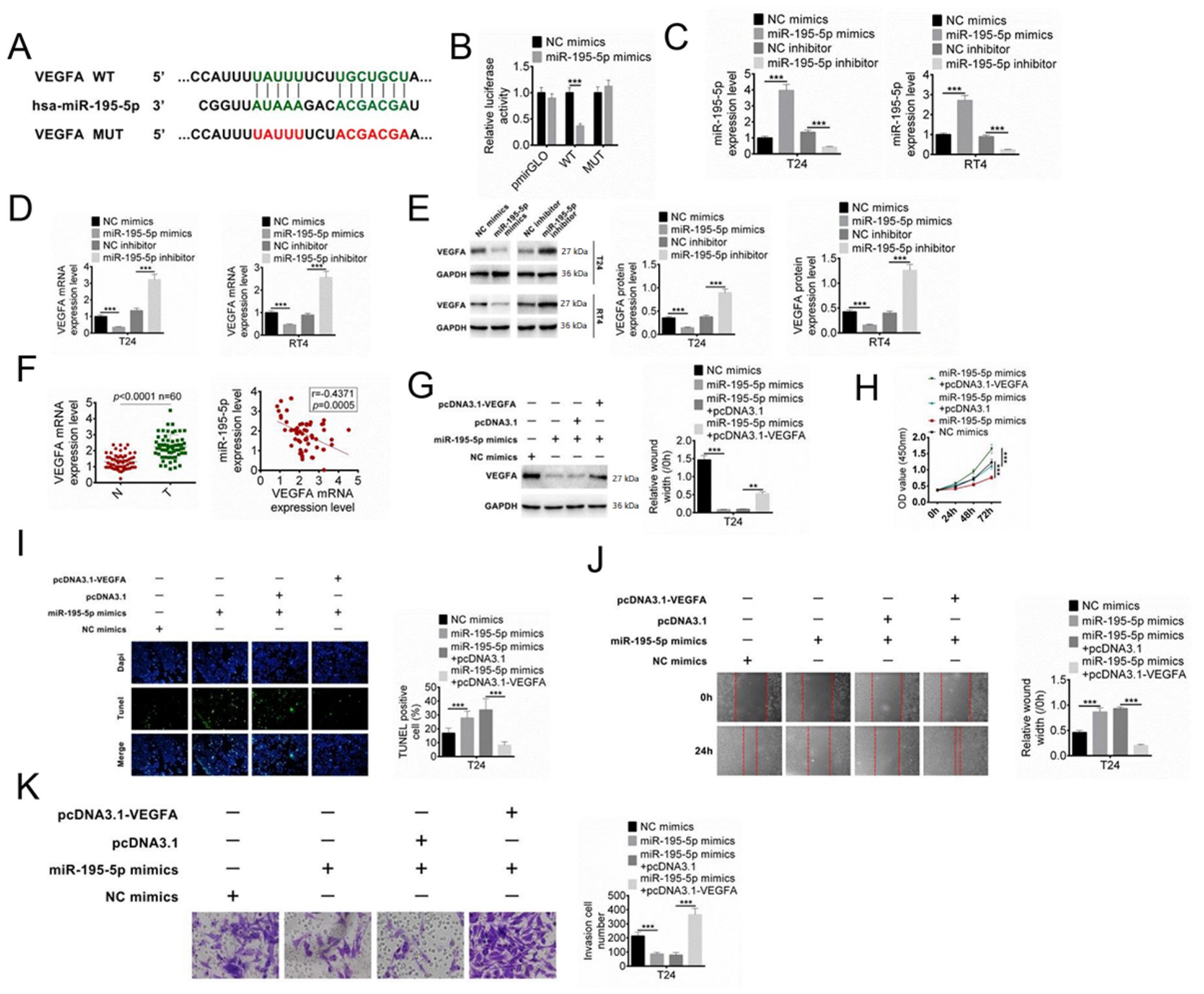

\section{Figure 5}

MiR-195-5p targets VEGFA and inhibits proliferation, migration, and invasion of BC cells. (A) The target of miR-195-5p was predicted by Targetscan. (B) The binding of miR-195-5p and VEGFA was verified by dualluciferase reporter assay. (C) The expression efficiency of miR-195-5p by miR-195-5p mimics and miR195-5p inhibitor. (D-E) The mRNA (D) and protein (E) expression of VEGFA in T24 and RT4 cells were induced by miR-195-5p mimics and miR-195-5p inhibitor. (F) The effect of pcDNA3.1-VEGFA and miR195-5p mimics on the VEGFA protein expression was determined by western blot. (G) The VEGFA expression in BC tissues and the relationship of VEGFA and miR-195-5p expression. VEGFA was highly expressed in the BC tissues and negatively correlated with the miR-195-5p expression. (H-K) MiR-195-5p mimics induced apoptosis $(\mathrm{I})$ and inhibited proliferation $(\mathrm{H})$, migration $(\mathrm{J})$, and invasion $(\mathrm{K})$, while these effects were abrogated by pcDNA3.1-VEGFA. Data were expressed as the mean $\pm S D, n=3$. ${ }^{*}<0.05$, ${ }^{\star *} P$ $<0.01, * \star * P<0.001$. 


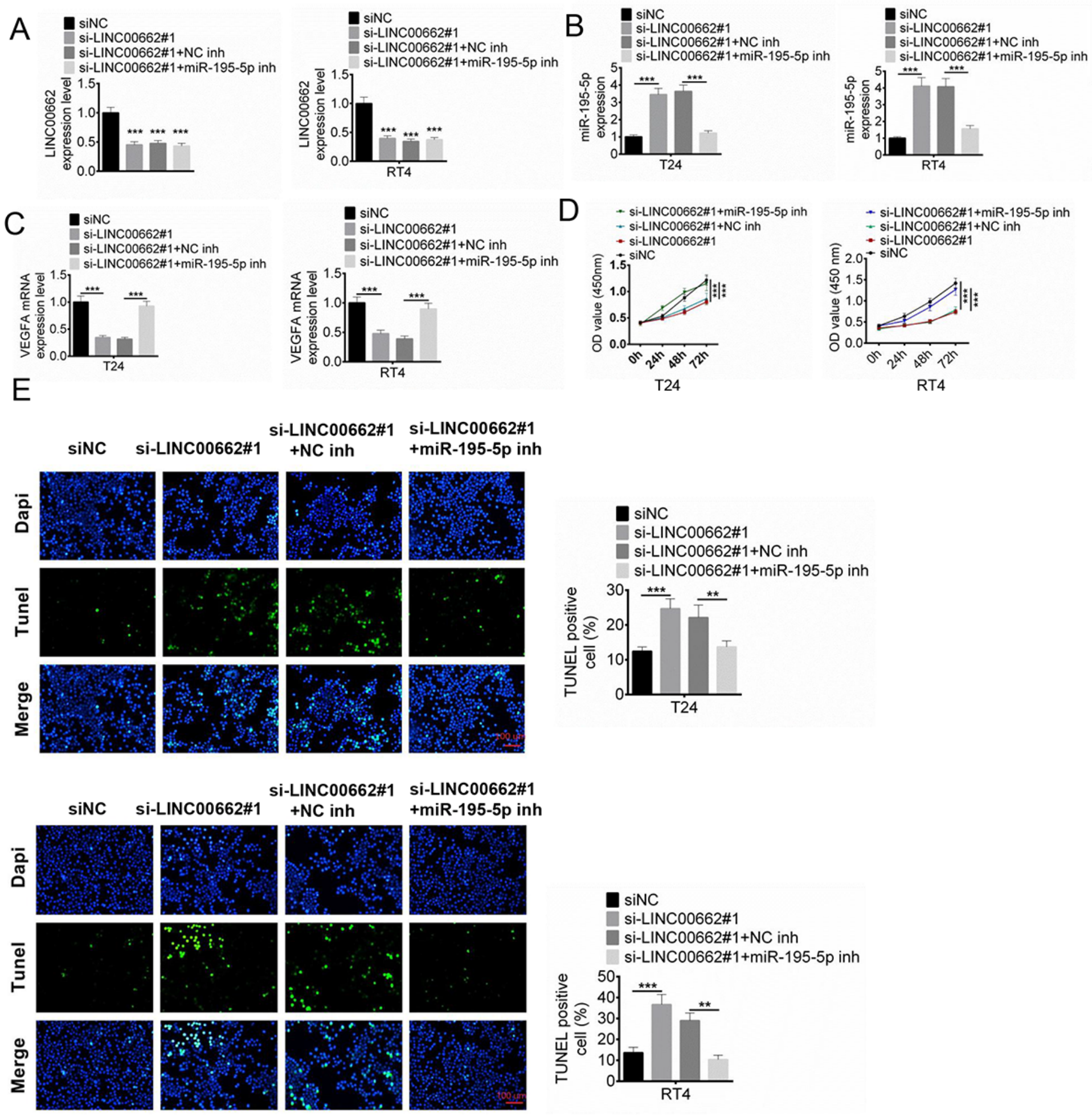

Figure 6

Effect of LINC00662 and miR-195-5p on proliferation, and apoptosis. (A-C) BC cells were transfected with siNC, siNC + si-LINC00662, si-LINC00662 + NC inhibitor, or si-LINC00662 + miR-195-5p inhibitor. LINC00662 expression (A), miR-miR-195-5p expression (B), and VEGFA mRNA expression (C) were examined by qRT-PCR assay. (D) Cell proliferation of BC cells was identified by CCK8 kit. (E) Cell 
apoptosis was examined by TUNEL assay. Data were expressed as the mean $\pm S D, n=3 .{ }^{*} P<0.05$, **P< $0.01, * * * P<0.001$.

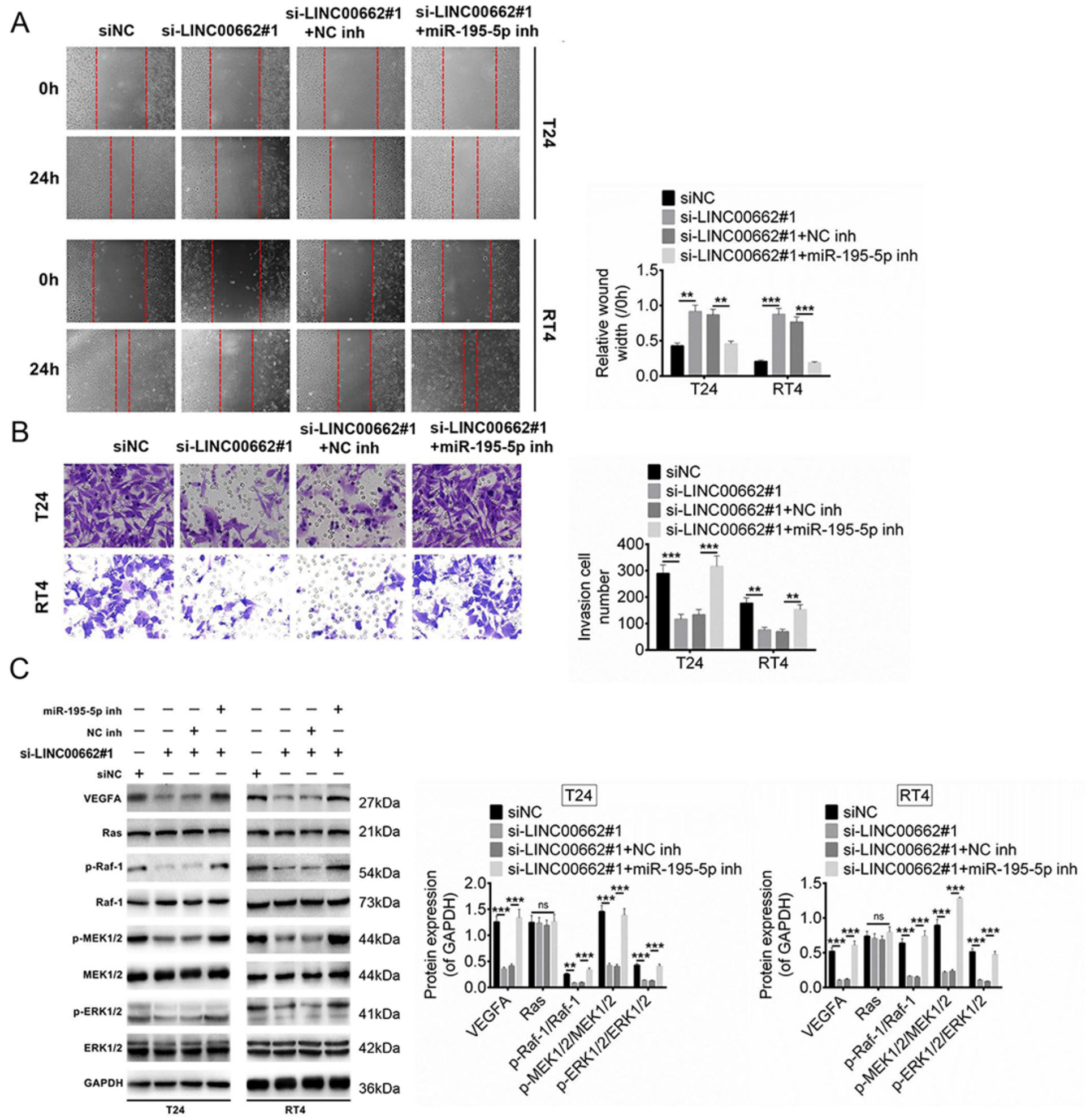

\section{Figure 7}

Effect of LINC00662 and miR-195-5p on migration, and invasion of BC cells and phosphorylation of VEGFA, Ras, MEK1/2, ERK1/2. (A) Cell migration was detected by wound healing assay. (B) Cell migration was identified by transwell assay. (C) The effect of sh-LINC00662 and miR-195-5p inhibitor on protein 
expression and phosphorylation levels of VEGFA and Ras/ERK signaling molecules was evaluated by western blot. Data were expressed as the mean $\pm S D, n=3 .{ }^{*} P<0.05, * \star P<0.01,{ }^{\star * \star} P<0.001$. 